Math. Model. Nat. Phenom.

Vol. 5, No. 7, 2010, pp. 116-121

DOI: $10.1051 / \mathrm{mmnp} / 20105719$

\title{
Integer Programming Formulation of the Bilevel Knapsack Problem
}

\author{
R. Mansi, S. Hanafi* and L. Brotcorne \\ LAMIH-SIADE, University of Valenciennes, France
}

\begin{abstract}
The Bilevel Knapsack Problem (BKP) is a hierarchical optimization problem in which the feasible set is determined by the set of optimal solutions of parametric Knapsack Problem. In this paper, we propose two stages exact method for solving the BKP. In the first stage, a dynamic programming algorithm is used to compute the set of reactions of the follower. The second stage consists in solving an integer program reformulation of BKP. We show that the integer program reformulation is equivalent to the BKP. Numerical results show the efficiency of our method compared with those obtained by the algorithm of Moore and Bard.
\end{abstract}

Key words: Bilevel programming, Knapsack problem, dynamic programming, branch-and-bound AMS subject classification: 65K05, 90C30

\section{Introduction}

The Bilevel Multidimensional Knapsack Problem (BKP) is a hierarchical optimization problem in which the feasible set is determined by the set of optimal solutions of parametric Knapsack Problem (KP). The BKP can be formulated as follows:

(BKP) Maximize $\quad f^{1}(x, y)=d^{1} x+d^{2} y$

subject to $B^{1} x+B^{2} y \leq b^{1}$,

$x \in \mathbb{N}^{n^{1}}$,

$y \in \operatorname{Argmax}\left\{f^{2}\left(y^{\prime}\right)=c^{2} y^{\prime}: a^{1} x+a^{2} y^{\prime} \leq b, y^{\prime} \in \mathbb{N}^{n^{2}}\right\}$,

\footnotetext{
${ }^{*}$ Corresponding author. E-mail: said.hanafi@univ-valenciennes.fr
} 
where the vectors $d^{1}$ and $a^{1}$ (respectively $a^{2}, c^{2}, d^{2}$ ) are of dimension $n^{1}\left(\right.$ resp. $n^{2}$ ). The vector $b^{1}$ is of dimension $m$. The coefficients of the matrices $B^{1}\left(m \times n^{1}\right)$ and $B^{2}\left(m \times n^{2}\right)$ are integers. We assume that the coefficients of the vectors $a^{1}$ and $a^{2}$ and the second member $b$ are positives integers. Vector $x$ (resp. $y$ ) of dimension $n^{1}$ (resp. $n^{2}$ ) represents the leader variables (resp. the follower). The function $f^{1}(x, y)$ (resp. $f^{2}(y)$ ) corresponds to the objective of the leader (resp. follower).

Bilevel programs have been the subject of extensive study both from a theoretical and a practical point of view (see [3], [6] for example). Several methods were proposed to solve linear bilevel problems with or without integer variables [4]. The particular structure of the bilevel knapsack problem with a continuous leader variable was studied by [5] and [1]. The main difficulties are due to the nonconvexity of its feasible region, which may result in an exponential number of local optima [2]. The nature of variables of bilevel mixed integer programming problem influences directly the existence of optimal solution. For example, bilevel mixed integer programming problems may not admit an optimal solution if there are continuous leader variables and integer variables for the follower [4]. In this paper, we propose an exact method for BKP which is decomposed into two phases. In the first one the dynamic programming algorithm is used to determine all possible reactions of the follower. In the second phase the result of the dynamic programming phase is used to provide an equivalent reformulation of BKP as an integer linear program. Then this integer linear problem is solved by a branch-and-bound algorithm.

\section{Dynamic programming}

In this phase, we consider only the integer knapsack problem of the follower by ignoring the resources $a^{1} x$ consumed by the leader:

$$
(K P) \quad \operatorname{Maximize}\left\{f^{2}(y)=c^{2} y: a^{2} y \leq b, y \in \mathbb{N}^{n^{2}}\right\} .
$$

The dynamic programming (DP) is a fundamental technique for solving optimization problems. The DP is also suitable if we want to solve the knapsack problem KP for all capacities $0, \ldots, b$. This is our goal in this phase since we try to obtain the set of the feasible solutions of the follower problem. Formally, the DP approach [7] consists to break down the problem KP in terms of its sub-problems for $k=1, \ldots, n^{2}$ and $\beta=0, \ldots, b$ :

$$
f_{k}^{2}(\beta)=\max \left\{\sum_{j=1}^{k} c_{j}^{2} y_{j}: \sum_{j=1}^{k} a_{j}^{2} y_{j} \leq \beta, y \in \mathbb{N}^{k}\right\} .
$$

The recurrence rules for computing $f_{k}^{2}(\beta)$ are:

$$
f_{k}^{2}(\beta)=\max \left\{f_{k-1}^{2}\left(\beta-h \times a_{k}^{2}\right): h=0, \ldots, u_{j}, \beta, y \geq h \times a_{k}^{2}\right\},
$$

for $k=2, \ldots, n^{2}$ and $\beta=0, \ldots, b$, where $u_{j}$ is an upper bound of the variable $y_{j}$. 


\section{Integer programming reformulation of the BKP}

The goal of the second phase is to reformulate the BKP problem as a linear integer program using the information resulted from the dynamic programming algorithm. To achieve this, we will link the leader action with the compatible reaction of the follower by respecting the optimality of the follower problem. More precisely, we propose to define explicitly a set of follower reactions for fixed $x$ in order to replace the follower optimization problem by the convex hull of these reactions. The follower's rational reaction set for fixed $x$, is

$$
P(x)=\operatorname{Argmax}\left\{f^{2}(y)=c^{2} y: a^{2} y \leq b-a^{1} x, y \in \mathbb{N}^{n^{2}}\right\} .
$$

To construct the follower's rational reaction set for fixed $x, P(x)$ we first compute the sensitivity intervals of the follower reactions, $y$, respecting the leader action. These sensitivity intervals are determined by the bounds of each interval $s^{l}$, which can be generated by the following rules:

$$
s^{1}=0, s^{l}=\min \left\{b-a^{1} x: f_{n^{2}}^{2}\left(b-a^{1} x\right)>f_{n^{2}}^{2}\left(s^{l-1}\right)\right\}, s^{p+1}=b+1,
$$

where $p=\max \left\{l: f_{n^{2}}^{2}(b)>f_{n^{2}}^{2}\left(s^{l-1}\right)\right\}$ is the number of intervals. The Inducible Region (IR), or the field on which the leader optimizes,

$$
I R(B K P)=\left\{(x, y) \in \mathbb{N}^{n^{1}} \times \mathbb{N}^{n^{2}}: B^{1} x+B^{2} y \leq b^{1}, a^{1} x+a^{2} y \leq b, y \in P(x)\right\} .
$$

From the definition of the inducible region of the bilevel knapsack problem $\operatorname{IR}(B K P)$, the BKP is equivalent to the following optimization problem:

$$
\left(B K P^{\prime}\right) \max \left\{f^{2}(y):(x, y) \in I R(B K P)\right\} \text {. }
$$

Finally, the BKP can be reformulated as the following integer program:

$$
\begin{array}{ll}
\text { MIP(BKP) } \quad \text { Maximize } \quad & f^{1}(x, y)=d^{1} x+d^{2} y \\
& B^{1} x+B^{2} y \leq b^{1}, \\
& a^{1} x+a^{2} y \leq b \\
& a^{1} x+\sum_{l=0}^{p} s^{l+1} z^{l} \geq b+1, \\
& c^{2} y=\sum_{l=0}^{p} f_{n^{2}}^{2} z^{l} \\
& \sum_{l=0}^{p} z^{l}=1 \\
& x \in \mathbb{N}^{n^{1}}, y \in \mathbb{N}^{n^{2}}, z \in\{0,1\}^{p+1} .
\end{array}
$$

For each fixed leader decision $x$, we should seek for the unique sensitivity interval $\left[s^{l}, s^{l+1}[\right.$ containing the resource $b-a^{2} x$. The binary variables $z^{l}$ are introduced for choosing the compatible 
interval. The reaction of the follower $y$ must be feasible for the leader constraints (3.2) and the follower constraints (3.3). The choice constraint (3.6) expresses that for each leader decision, only a unique sensitivity interval has to be chosen. The constraints (3.4) and (3.5) ensure that the accurate interval chosen corresponds to the optimal value of the follower problem. In other words the selected interval must be compatible with the leader decision $x$. The integer program $M I P(B K P)$ can now be solved exactly (if possible) using an exact algorithm. In our experiments this model is solved by the "Branch-and-Bound" of CPLEX software.

\section{Computational experiments}

In this section, we present initially a comparative study between the proposed algorithm called DBKP (Dynamic Programming for the BKP) and that of Moore and Bard (1990) [8]. Algorithms were developed in $\mathrm{C}++$ language and by using the solver CPLEX 11.1. The tests were carried out on a computer based on a processor Xeon 5160 of capacity $3 \mathrm{GHz}$ and $16 \mathrm{~GB}$ of RAM. Test cases are generated randomly by varying the number of variables $\left(n^{1}, n^{2}\right)$ and the number of leader constraints. The generation of the follower data was carried out by generator for the Knapsack problems according to a non correlated degree of correlation. We consider that the maximal size of the coefficients is 1000 . Note that all the variables of the problem are limited by the value 10. The results shown in Table 1 represent the average of 10 test cases. We present here the results obtained for the cases associated with $m^{1} \in\{5,10,15\}, n^{1} \in\{5,6,7,8,9,10\}$ and $n^{2} \in\{10,15,20,25,30\}$.

The significant execution times of the algorithm MB can be justified by the fact that problems which have to be solved at each node are NP-Hard. Moreover, the upper bound provided at each node is not strong. Consequently, the number of nodes traversed by the branch and bound increases very quickly with the size of the test cases. The DBKP algorithm is very effective to solve all the est cases of Table 1. The application of the dynamic programming on the problem of second level with maximum 30 variables is instantaneous. Note that our algorithm solves one mixed linear program where the MB algorithm solves several mixed linear programs at each node.

\section{Conclusion}

We proposed a new exact approach to solve the Bilevel Knapsack problem in which the leader controls a set of integer variables and the follower solves an integer parameterized knapsack problem. Our method is decomposed into two phases: the first phase is based on the dynamic programming, in which the recurrence rules take into account only the follower problem. Consequently, in the second phase we reformulated BKP problem as a linear integer program. Our numerical experiments showed that our algorithm is significantly more powerful than the algorithms proposed in the literature, Moore and Bard [8]. 


\begin{tabular}{|c|c|c|c|c|c|c|c|c|c|c|c|c|c|c|}
\hline$m^{1}$ & $n^{2}$ & $n^{1}$ & DBKP & MB & $m^{1}$ & $n^{2}$ & $n^{1}$ & DBKP & MB & $m^{1}$ & $n^{2}$ & $n^{1}$ & DBKP & MB \\
\hline \multirow{30}{*}{5} & \multirow{6}{*}{10} & 5 & 0.01 & 0.21 & \multirow{30}{*}{10} & \multirow{6}{*}{10} & 5 & 0.01 & 0.18 & \multirow{30}{*}{15} & \multirow{6}{*}{10} & 5 & 0.01 & 0.40 \\
\hline & & 6 & 0.01 & 2.77 & & & 6 & 0.01 & 0.04 & & & 6 & 0.01 & 2.34 \\
\hline & & 7 & 0.01 & 0.01 & & & 7 & 0.01 & 3.39 & & & 7 & 0.01 & 2.53 \\
\hline & & 8 & 0.01 & 6.18 & & & 8 & 0.01 & 4.39 & & & 8 & 0.02 & 8.90 \\
\hline & & 9 & 0.02 & 40.01 & & & 9 & 0.00 & 0.01 & & & 9 & 0.01 & 6.53 \\
\hline & & 10 & 0.01 & 0.01 & & & 10 & 0.00 & 0.49 & & & 10 & 0.01 & 0.03 \\
\hline & \multirow{6}{*}{15} & 5 & 0.01 & 0.11 & & \multirow{6}{*}{15} & 5 & 0.01 & 18.36 & & \multirow{6}{*}{15} & 5 & 0.01 & 12.69 \\
\hline & & 6 & 0.01 & 14.43 & & & 6 & 0.01 & 10.84 & & & 6 & 0.01 & 3.87 \\
\hline & & 7 & 0.01 & 40.32 & & & 7 & 0.00 & 0.01 & & & 7 & 0.01 & 6.15 \\
\hline & & 8 & 0.02 & 105.41 & & & 8 & 0.01 & 24.97 & & & 8 & 0.01 & 4.91 \\
\hline & & 9 & 0.01 & 27.80 & & & 9 & 0.02 & 18.40 & & & 9 & 0.01 & 48.15 \\
\hline & & 10 & 0.05 & 26.68 & & & 10 & 0.00 & 0.41 & & & 10 & 0.02 & 21.04 \\
\hline & \multirow{6}{*}{20} & 5 & 0.01 & 2.81 & & \multirow{6}{*}{20} & 5 & 0.01 & 4.32 & & \multirow{6}{*}{20} & 5 & 0.01 & 0.12 \\
\hline & & 6 & 0.01 & 6.08 & & & 6 & 0.02 & 33.63 & & & 6 & 0.04 & 167.42 \\
\hline & & 7 & 0.06 & 821.46 & & & 7 & 0.01 & 0.01 & & & 7 & 0.01 & 6.67 \\
\hline & & 8 & 0.02 & 10.22 & & & 8 & 0.11 & 639.46 & & & 8 & 0.12 & 920.60 \\
\hline & & 9 & 0.01 & 9.49 & & & 9 & 0.01 & 1.23 & & & 9 & 0.01 & 64.56 \\
\hline & & 10 & 0.01 & 0.08 & & & 10 & 0.03 & 2663.35 & & & 10 & 0.24 & 1671.82 \\
\hline & \multirow{6}{*}{25} & 5 & 0.01 & 7.49 & & \multirow{6}{*}{25} & 5 & 0.01 & 0.01 & & \multirow{6}{*}{25} & 5 & 0.01 & 24.89 \\
\hline & & 6 & 0.01 & 26.33 & & & 6 & 0.03 & 250.34 & & & 6 & 0.02 & 386.34 \\
\hline & & 7 & 0.02 & 4.62 & & & 7 & 0.08 & 2096.18 & & & 7 & 0.15 & 336.00 \\
\hline & & 8 & 0.08 & 728.79 & & & 8 & 0.11 & 64.36 & & & 8 & 0.01 & 25.10 \\
\hline & & 9 & 0.03 & 18.44 & & & 9 & 0.02 & 201.19 & & & 9 & 0.11 & 1435.86 \\
\hline & & 10 & 0.04 & 834.47 & & & 10 & 0.06 & 10233.96 & & & 10 & 0.13 & 3373.12 \\
\hline & \multirow{6}{*}{30} & 5 & 0.07 & 3374.58 & & \multirow{6}{*}{30} & 5 & 0.01 & 0.15 & & \multirow{6}{*}{30} & 5 & 0.03 & 70.50 \\
\hline & & 6 & 0.12 & 9152.25 & & & 6 & 0.02 & 6.11 & & & 6 & 0.02 & 33.15 \\
\hline & & 7 & 0.02 & 10.11 & & & 7 & 0.03 & 14.06 & & & 7 & 0.07 & 1630.47 \\
\hline & & 8 & 0.14 & 14874.49 & & & 8 & 0.14 & 1585.35 & & & 8 & 0.14 & 21658.78 \\
\hline & & 9 & 0.01 & 0.08 & & & 9 & 0.11 & 12652.31 & & & 9 & 0.02 & 415.01 \\
\hline & & 10 & 0.01 & 0.70 & & & 10 & 0.06 & 163.91 & & & 10 & 0.02 & 247.63 \\
\hline
\end{tabular}

Table 1: Comparison between DBKP and MB algorithms.

\section{Acknowledgements}

The present research work has been supported by International Campus on Safety and Intermodality in Transportation the Nord-Pas-de-Calais region, the European Community, the Regional Delegation for Research and Technology, the Ministry of Higher Education and Research, and the National Center for Scientific Research. The authors gratefully acknowledge the support of these institutions.

\section{References}

[1] L. Brotcorne, S. Hanafi, R. Mansi. A dynamic programming algorithm for the bilevel knapsack problem. Operations Research Letters, 37 (2009), No. 3, 215-218.

[2] P. Calamai, L. Vicente. Generating linear and linear-quadratic Bilevel programming problems. SIAM Journal on Scientific and Statistical Computing, 14 (1993), 770-782.

[3] B. Colson, P. Marcotte, G. Savard. Bilevel programming, a survey. 4OR, 3 (2005), 87-107.

[4] S. Dempe. Foundation of Bilevel programming. Kluwer academic publishers, 2002. 
[5] S. Dempe, K. Richter. Bilevel programming with Knapsack constraints. Central European Newspaper of Operations Research, 8 (2000), 93-107.

[6] P. Hansen, B. Jaumard, G. Savard. New branch-and-bound rules for linear bilevel programming. SIAM Journal on Scientific and Statistical Computing, 13 (1992), 1194-1217.

[7] H. Kellerer, U. Pferschy, D. Pisinger. Knapsack problems. Springer-Verlag, 2004.

[8] J.T. Moore, J.F. Bard. The mixed integer linear Bilevel programming problem. Operations Research, 38 (1990), 911-921. 